\title{
Editorial
}

\section{Pharmacologic Treatment of Obesity}

\author{
Alfredo Halpern, ${ }^{1}$ Steven B. Heymsfield, ${ }^{2}$ and Luc Van Gaal ${ }^{3}$ \\ ${ }^{1}$ University of São Paulo, 05508 São Paulo, SP, Brazil \\ ${ }^{2}$ Pennington Biomedical Research Center, Baton Rouge, LA 70808, USA \\ ${ }^{3}$ University of Antwerp, 2650 Edegem/Antwerp, Belgium \\ Correspondence should be addressed to Alfredo Halpern, halpern@netpoint.com.br \\ Received 26 February 2012; Accepted 26 February 2012
}

Copyright (c) 2011 Alfredo Halpern et al. This is an open access article distributed under the Creative Commons Attribution License, which permits unrestricted use, distribution, and reproduction in any medium, provided the original work is properly cited.

The pharmacological treatment of obesity still faces many barriers. Notably, these include the disregard of obesity as a disease and the very limited treatment options for obesity. In this context, this special issue is of special importance as it focuses on several topics, related to this challenge.

After careful screening, review, and revision, we selected 26 articles which deal with a variety of aspects related to the pharmacological therapy of obesity. Topics covered include possible etiologies of the disease (e.g., A. Niijima's rat study and B. Bjorndal et al.'s research on adipogenesis), the role of diet as an anti-inflammatory mediator (studies of $\mathrm{B}$. Sears and C. Ricordi and W. R. Hamilton et al.), some new aspects of the drugs being used today, particularly orlistat (A. Hollywood and J. Ogden, J. Martin et al., and N. J. Perera et al. studies), and outlining the treatment of special conditions such as in children and adolescents (L. Iughett et al.), polycystic ovary syndrome (H. Kahal et al.), and diabetes mellitus (V. Cheng and S. R. Kashyap). Additional reports include reviews on the pharmacological treatment of obesity in the present and future (M. Glandt and I. Raz and L. L. Ioannides-Demos et al.).

To conclude, this edition of the journal describing advances in the pharmacological treatment of obesity will provide those in the field with new and useful insights on obesity pathogenesis and management.

Alfredo Halpern

Steven B. Heymsfield

Luc Van Gaal 


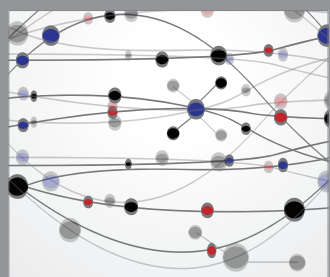

The Scientific World Journal
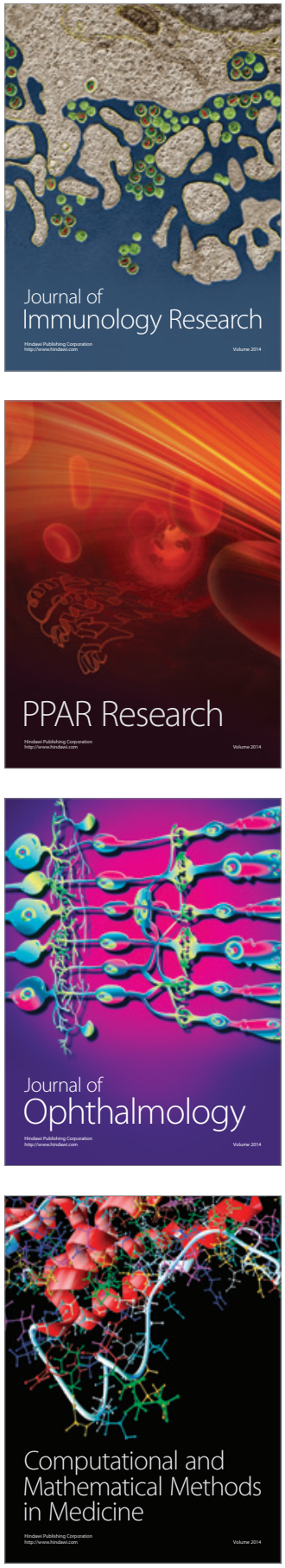

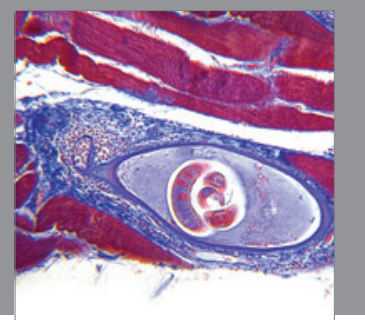

Gastroenterology

Research and Practice
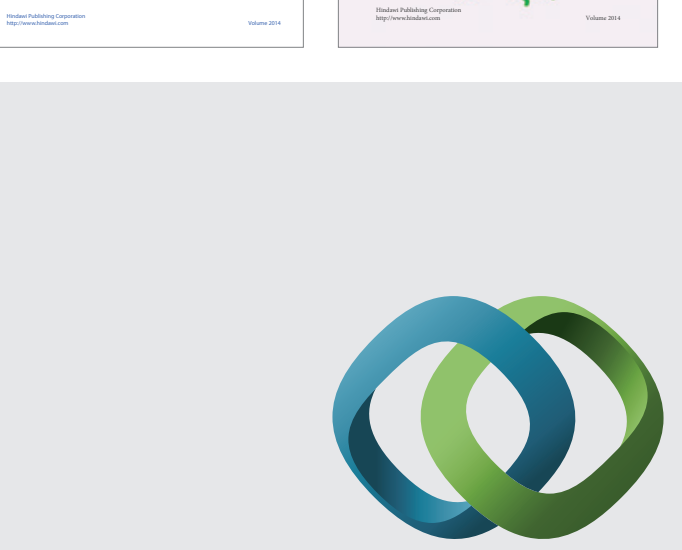

\section{Hindawi}

Submit your manuscripts at

http://www.hindawi.com
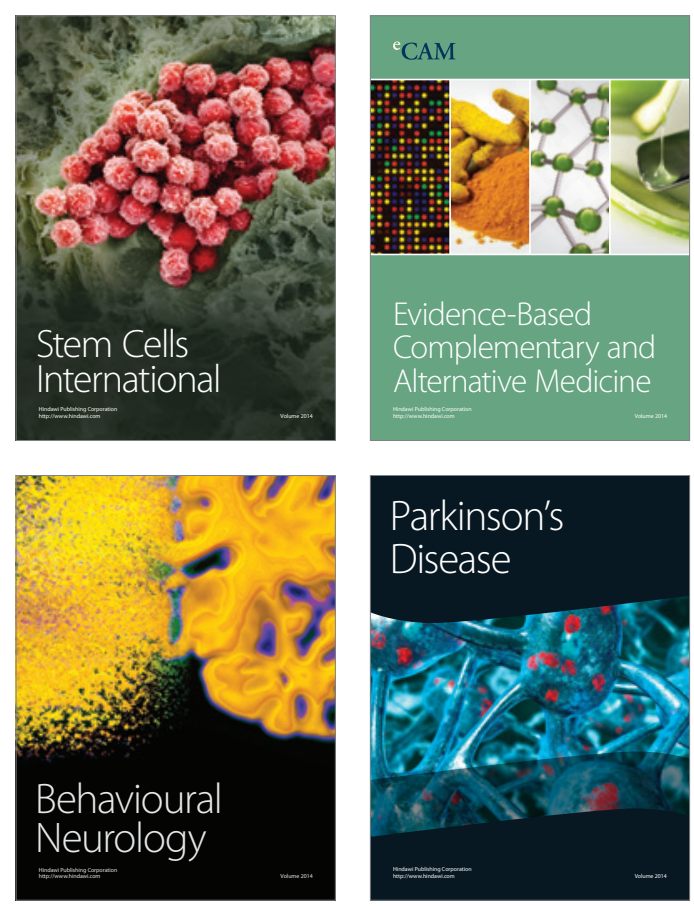

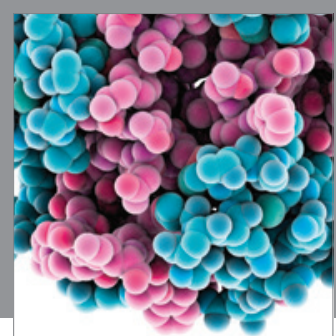

Journal of
Diabetes Research

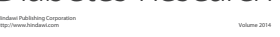

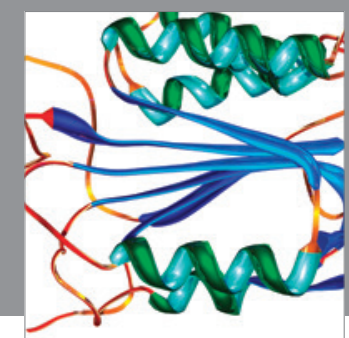

Disease Markers
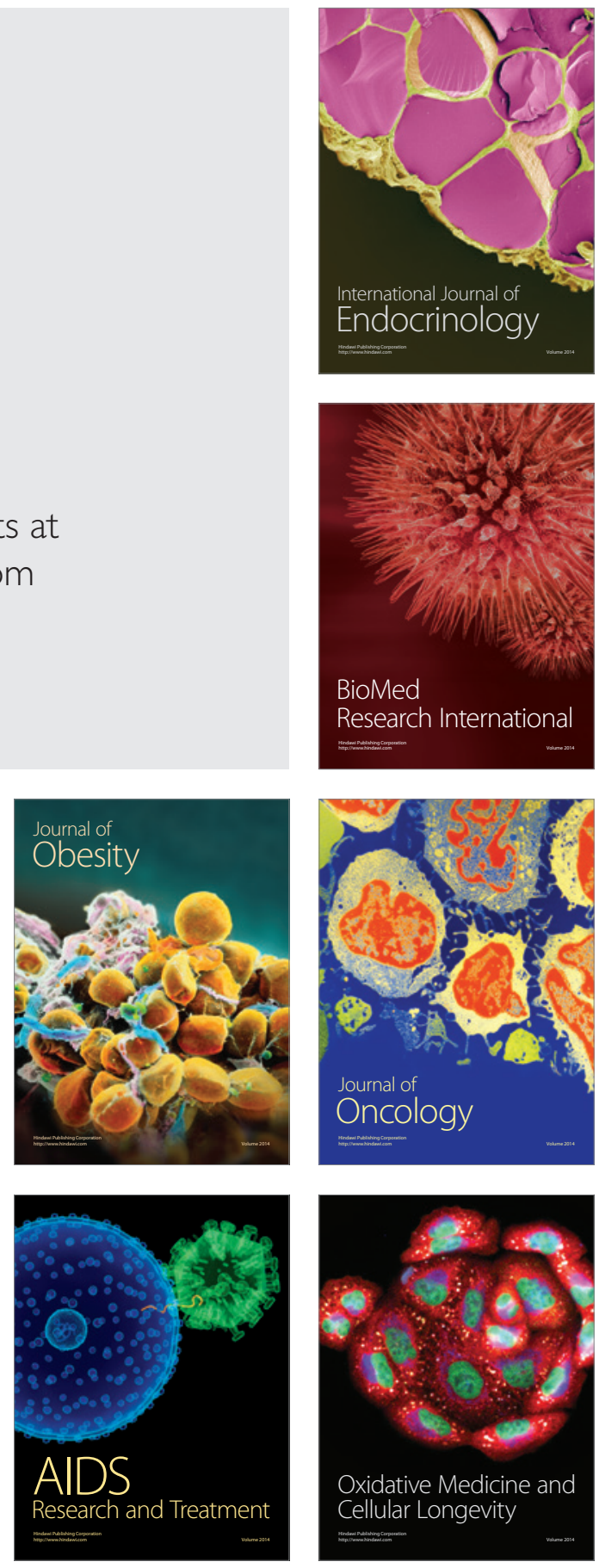